\title{
Unconditional uniqueness in the charge class for the Dirac-Klein-Gordon equations in two space dimensions
}

\author{
Sigmund Selberg and Achenef Tesfahun
}

\begin{abstract}
Recently, Grünrock and Pecher proved global well-posedness of the $2 \mathrm{~d}$ Dirac-Klein-Gordon equations given initial data for the spinor and scalar fields in $H^{s}$ and $H^{s+1 / 2} \times H^{s-1 / 2}$, respectively, where $s \geq 0$, but uniqueness was only known in a contraction space of Bourgain type, strictly smaller than the natural solution space $C\left([0, T] ; H^{s} \times H^{s+1 / 2} \times\right.$ $\left.H^{s-1 / 2}\right)$. Here we prove uniqueness in the latter space for $s \geq 0$. This improves a recent result of Pecher, where the range $s>1 / 30$ was covered.
\end{abstract}

Mathematics Subject Classification (2010). 35Q40; 35L70.

Keywords. Dirac-Klein-Gordon equations, Uniqueness, Null structure, Bilinear space-time estimates.

\section{Introduction}

We consider the Dirac-Klein-Gordon system (DKG) in two space dimensions, which reads

$$
\left\{\begin{array}{l}
-i\left(\partial_{t}+\alpha \cdot \nabla\right) \psi=-M \beta \psi+\phi \beta \psi, \\
\left(-\square+m^{2}\right) \phi=\langle\beta \psi, \psi\rangle, \quad\left(\square=-\partial_{t}^{2}+\Delta\right)
\end{array}\right.
$$

with initial data

$$
\left.\psi\right|_{t=0}=\psi_{0} \in H^{s},\left.\quad \phi\right|_{t=0}=\phi_{0} \in H^{r},\left.\quad \partial_{t} \phi\right|_{t=0}=\phi_{1} \in H^{r-1},
$$

where $\psi(t, x)$ is the Dirac spinor, regarded as a column vector in $\mathbb{C}^{2}$, and $\phi(t, x)$ is real-valued. Here $t \in \mathbb{R}, x \in \mathbb{R}^{2} ; M, m \geq 0$ are constants; $\nabla=$ $\left(\partial_{x_{1}}, \partial_{x_{2}}\right) ;\langle u, v\rangle:=\langle u, v\rangle_{\mathbb{C}^{2}}=v^{\dagger} u$ for column vectors $u, v \in \mathbb{C}^{2}$, where $v^{\dagger}$ is the complex conjugate transpose of $v ; H^{s}=(1+\sqrt{-\Delta})^{-s} L^{2}\left(\mathbb{R}^{2}\right)$ is the standard Sobolev space of order $s$. The Dirac matrices $\alpha^{j}, \beta$ satisfy

$$
\beta^{\dagger}=\beta, \quad\left(\alpha^{j}\right)^{\dagger}=\alpha^{j}, \quad \beta^{2}=\left(\alpha^{j}\right)^{2}=I, \quad \alpha^{j} \beta+\beta \alpha^{j}=0 .
$$


A particular representation is

$$
\alpha^{1}=\left(\begin{array}{ll}
0 & 1 \\
1 & 0
\end{array}\right), \quad \alpha^{2}=\left(\begin{array}{cc}
0 & -i \\
i & 0
\end{array}\right), \quad \beta=\left(\begin{array}{cc}
1 & 0 \\
0 & -1
\end{array}\right) .
$$

Local well-posedness of this problem with low-regularity data was first studied in [1]. In [3], D'Ancona et al. proved the existence of a local solution

$$
\left(\psi, \phi, \partial_{t} \phi\right) \in C\left([0, T] ; H^{s} \times H^{r} \times H^{r-1}\right)
$$

for the Cauchy problem (1.1), (1.2) whenever

$$
s>-\frac{1}{5}, \quad \max \left(\frac{1}{4}+\frac{|s|}{2}, s\right)<r<\min \left(\frac{3}{4}+2 s, 1+s\right),
$$

but uniqueness was only obtained in the contraction space, which is strictly smaller than the natural solution space $C\left([0, T] ; H^{s} \times H^{r} \times H^{r-1}\right)$. The question therefore arises whether uniqueness holds in the latter space; if this is the case, one says that unconditional uniqueness holds at the regularity $(s, r)$. The question of unconditional uniqueness is especially interesting for $s \geq 0$ and $r=s+1 / 2$, since for that range it was proved by Grünrock and Pecher [5] that the local solution extends globally in time. Recently, Pecher [6] proved unconditional uniqueness for $s>1 / 30, r=s+1 / 2$. In this paper we improve this to $s \geq 0$.

Theorem 1.1. If $s \geq 0$, uniqueness of the solution to (1.1), (1.2) holds in

$$
\left(\psi, \phi, \partial_{t} \phi\right) \in C\left([0, T] ; H^{s} \times H^{s+1 / 2} \times H^{s-1 / 2}\right) .
$$

To prove this we rely on the null structure of DKG, found in [3], and on bilinear space-time estimates of Klainerman-Machedon type proved in [4]. Using also an idea of Zhou [7] we iteratively improve the known regularity of the solution, until we reach a space where uniqueness is known by the results in $[3]$.

Some notation: In estimates we shall use $X \lesssim Y$ as shorthand for $X \leq$ $C Y$, where $C \gg 1$ is a constant. We write $X \approx Y$ for $X \lesssim Y \lesssim X$. Throughout, $\varepsilon$ is understood to be a sufficiently small positive number. The space-time Fourier transform of a function $u(t, x)$ is denoted $\mathcal{F} u(\tau, \xi)=\widetilde{u}(\tau, \xi)$.

\section{Null structure and bilinear estimates}

To uncover the null structure of the system we follow [3] and use the Dirac projections

$$
P_{ \pm}(\xi)=\frac{1}{2}\left(I \pm \frac{\xi}{|\xi|} \cdot \alpha\right)
$$

to split $\psi=\psi_{+}+\psi_{-}$, where $\psi_{ \pm}=P_{ \pm}(D) \psi$. Here $D=-i \nabla$, which has Fourier symbol $\xi$. Applying $P_{ \pm}(D)$ to the Dirac equation in (1.1), and using the identities $\alpha \cdot D=|D| P_{+}(D)-|D| P_{-}(D), P_{ \pm}^{2}(D)=P_{ \pm}(D), P_{ \pm}(D) P_{\mp}(D)=0$ and $P_{ \pm}(D) \beta=\beta P_{\mp}(D)$, the Dirac equation becomes

$$
\left(-i \partial_{t} \pm|D|\right) \psi_{ \pm}=-M \beta \psi_{\mp}+P_{ \pm}(D)(\phi \beta \psi) .
$$


On the other hand, splitting $\phi=\phi_{+}+\phi_{-}$, where

$$
\phi_{ \pm}=\frac{1}{2}\left(\phi \pm i\langle D\rangle_{m}^{-1} \phi_{t}\right), \quad\langle D\rangle_{m}=\sqrt{m^{2}+|D|^{2}}
$$

the Klein-Gordon part of the system becomes

$$
\left(-i \partial_{t} \pm\langle D\rangle_{m}\right) \phi_{ \pm}=\mp\left(2\langle D\rangle_{m}\right)^{-1}\langle\beta \psi, \psi\rangle .
$$

Thus, the DKG system has been rewritten as

$$
\left\{\begin{array}{l}
\left(-i \partial_{t} \pm|D|\right) \psi_{ \pm}=-M \beta \psi_{\mp}+P_{ \pm}(D)(\phi \beta \psi) \\
\left(-i \partial_{t} \pm\langle D\rangle_{m}\right) \phi_{ \pm}=\mp\left(2\langle D\rangle_{m}\right)^{-1}\langle\beta \psi, \psi\rangle
\end{array}\right.
$$

with initial data

$$
\psi_{ \pm}(0)=P_{ \pm}(D) \psi_{0} \in H^{s}, \quad \phi_{ \pm}(0)=\frac{1}{2}\left(\phi_{0} \pm i\langle D\rangle_{m}^{-1} \phi_{1}\right) \in H^{s+\frac{1}{2}}
$$

Theorem 1.1 then reduces to the following.

Theorem 2.1. If $s \geq 0$, uniqueness of the solution to (2.1), (2.2) holds in

$$
\left(\psi_{ \pm}, \phi_{ \pm}\right) \in C\left([0, T] ; H^{s} \times H^{s+1 / 2}\right) .
$$

The null structure in the second equation in (2.1) is quantified by the following estimate from [3]: For Schwartz functions $\psi, \psi^{\prime}: \mathbb{R}^{1+2} \rightarrow \mathbb{C}^{2}$ and independent signs $\pm_{1}$ and $\pm_{2}$,

$$
\begin{aligned}
& \left|\mathcal{F}\left\langle\beta P_{ \pm_{1}}(D) \psi, P_{ \pm_{2}}(D) \psi^{\prime}\right\rangle(\tau, \xi)\right| \\
& \quad \lesssim \int_{\mathbb{R}^{1+2}} \theta\left( \pm_{1} \eta, \pm_{2}(\eta-\xi)\right)|\widetilde{\psi}(\lambda, \eta)|\left|\widetilde{\psi}^{\prime}(\lambda-\tau, \eta-\xi)\right| d \lambda d \eta
\end{aligned}
$$

where $\theta(\xi, \eta)$ denotes the angle between nonzero $\xi, \eta \in \mathbb{R}^{2}$. The null structure in the first equation in (2.1) is seen to be of the same type via a duality argument (see the next section). To exploit this structure we need the following estimate:

Lemma 2.2. Let $a, b, c \in\left[0, \frac{1}{2}\right]$. For all signs $\left( \pm_{1}, \pm_{2}\right)$, all $\lambda, \mu \in \mathbb{R}$ and all nonzero $\eta, \zeta \in \mathbb{R}^{2}$,

$$
\theta\left( \pm_{1} \eta, \pm_{2} \zeta\right) \lesssim\left(\frac{\langle|\lambda-\mu|-|\eta-\zeta|\rangle}{\min (\langle\eta\rangle,\langle\zeta\rangle)}\right)^{a}+\left(\frac{\left\langle\lambda \pm_{1}|\eta|\right\rangle}{\min (\langle\eta\rangle,\langle\zeta\rangle)}\right)^{b}+\left(\frac{\left\langle\mu \pm_{2}|\zeta|\right\rangle}{\min (\langle\eta\rangle,\langle\zeta\rangle)}\right)^{c}
$$

Proof. In [2, Section 5.1] it is shown that this holds when $a=b=c=1 / 2$, and since $\theta\left( \pm_{1} \eta, \pm_{2} \zeta\right) \lesssim 1$ the lemma follows.

Next, we define some function spaces.

We remark that the symbols $\tau \pm|\xi|$ and $\tau \pm\langle\xi\rangle_{m}$ associated with the operators $-i \partial_{t} \pm|D|$ and $-i \partial_{t} \pm\langle D\rangle_{m}$ appearing in (2.1) are comparable, in the sense that $\langle\tau \pm|\xi|\rangle \approx\left\langle\tau \pm\langle\xi\rangle_{m}\right\rangle$, where $\langle\cdot\rangle_{m}=\sqrt{m^{2}+|\cdot|^{2}}$. Hence, the $X^{s, b}$ spaces corresponding to the two operators are in fact equivalent. Specifically, 
the space $X_{ \pm}^{s, b}$ in question is defined (given $s, b \in \mathbb{R}$ ) as the completion of $\mathcal{S}\left(\mathbb{R}^{1+2}\right)$ with respect to the norm

$$
\|u\|_{X_{ \pm}^{s, b}}=\left\|\langle\xi\rangle^{s}\langle\tau \pm|\xi|\rangle^{b} \widetilde{u}(\tau, \xi)\right\|_{L_{\tau, \xi}^{2}} .
$$

We also need the wave-Sobolev space $H^{s, b}$ with norm

$$
\|u\|_{H^{s, b}}=\left\|\langle\xi\rangle^{s}\langle|\tau|-|\xi|\rangle^{b} \widetilde{u}(\tau, \xi)\right\|_{L_{\tau, \xi}^{2}} .
$$

For $T>0$, let $X_{ \pm}^{s, b}\left(S_{T}\right)$ and $H^{s, b}\left(S_{T}\right)$ be the respective restriction spaces to the slab $S_{T}=(0, T) \times \mathbb{R}^{2}$.

Clearly, for $b \geq 0$,

$$
\|u\|_{H^{s, b}} \leq\|u\|_{X_{ \pm}^{s, b}} .
$$

We also recall that for $b>1 / 2, H^{s, b}$ is a proper subspace of $C\left(\mathbb{R} ; H^{s}\right)$, and the inclusion is continuous. Thus,

$$
X_{ \pm}^{s, b} \subset H^{s, b} \subset C\left(\mathbb{R}, H^{s}\right) \text { for } b>1 / 2 .
$$

Moreover, it is well known that the initial value problem

$$
\left(-i \partial_{t} \pm|D|\right) u=F \in X_{ \pm}^{s, b-1},\left.\quad u\right|_{t=0}=f \in H^{s}
$$

for any $s \in \mathbb{R}$ and $b>\frac{1}{2}$, has a unique solution $u \in C\left([0, T] ; H^{s}\right)$, and $u$ satisfies

$$
\|u\|_{X_{ \pm}^{s, b}\left(S_{T}\right)} \leq C\left(\|f\|_{H^{s}}+\|F\|_{X_{ \pm}^{s, b-1}\left(S_{T}\right)}\right),
$$

where $C$ depends on $b$ and $T$. See, e.g., [3].

The final key ingredient that we need for the proof of uniqueness is the following product estimate for the spaces $H^{s, b}$.

Theorem 2.3. Assume

$$
\begin{aligned}
& b_{0} \leq 0<b_{1}, b_{2} \\
& b_{0}+b_{1}+b_{2}>\frac{1}{2} \\
& b_{0}+b_{1}>0, \quad b_{0}+b_{2}>0 \\
& s_{0}+s_{1}+s_{2}>\frac{3}{2}-\left(b_{0}+b_{1}+b_{2}\right) \\
& s_{0}+s_{1}+s_{2}>1-\left(b_{0}+b_{1}\right) \\
& s_{0}+s_{1}+s_{2}>1-\left(b_{0}+b_{2}\right) \\
& s_{0}+s_{1}+s_{2}>\frac{1}{2}-b_{0} \\
& s_{0}+s_{1}+s_{2}>\frac{3}{4} \\
& s_{0}+b_{0}+2\left(s_{1}+s_{2}\right)>1 \\
& s_{1}+s_{2} \geq-b_{0}, \quad s_{0}+s_{2} \geq 0, \quad s_{0}+s_{1} \geq 0 .
\end{aligned}
$$

Then the product estimate

$$
\|u v\|_{H^{-s_{0},-b_{0}}} \lesssim\|u\|_{H^{s_{1}, b_{1}}}\|v\|_{H^{s_{2}, b_{2}}}
$$


holds for all $u, v \in \mathcal{S}\left(\mathbb{R}^{1+2}\right)$.

This follows from Theorems 4.1 and 6.1 in [4].

\section{Proof of uniqueness}

We now prove Theorem 2.1. Without loss of generality take $s=0$. From [3] it is known that the solution is unique in the iteration space

$$
\left(\psi_{ \pm}, \phi_{ \pm}\right) \in X_{ \pm}^{-\frac{5}{32}-, \frac{1}{2}+}\left(S_{T}\right) \times X_{ \pm}^{\frac{11}{32}-, \frac{1}{2}+}\left(S_{T}\right),
$$

where we use the notation $a \pm:=a \pm \delta$ for sufficiently small $\delta>0$. Thus, it suffices to show that if

$$
\left(\psi_{ \pm}, \phi_{ \pm}\right) \in C\left([0, T], L^{2} \times H^{\frac{1}{2}}\right)
$$

is a solution of $(2.1),(2.2)$, then

$$
\left(\psi_{ \pm}, \phi_{ \pm}\right) \in X_{ \pm}^{-\frac{5}{32}-, \frac{1}{2}+}\left(S_{T}\right) \times X_{ \pm}^{\frac{11}{32}-, \frac{1}{2}+}\left(S_{T}\right) .
$$

To this end, we split $\psi_{ \pm}$as

$$
\psi_{ \pm}=\psi_{ \pm}^{\mathrm{h}}+\psi_{ \pm}^{\mathrm{l}}+\Psi_{ \pm}
$$

where $\psi_{ \pm}^{\mathrm{h}}$ is the homogeneous part while $\psi_{ \pm}^{\mathrm{l}}$ and $\Psi_{ \pm}$are the inhomogeneous parts corresponding to the linear and bilinear terms, respectively, in the righthand side of the first equation in (2.1). Similarly, we split $\phi_{ \pm}$as

$$
\phi_{ \pm}=\phi_{ \pm}^{\mathrm{h}}+\Phi_{ \pm}
$$

where $\phi_{ \pm}^{\mathrm{h}}$ and $\Phi_{ \pm}$are the homogeneous and inhomogeneous parts of $\phi_{ \pm}$.

First note that by (2.6) and assumption (3.1),

$$
\psi_{ \pm}^{\mathrm{h}} \in X_{ \pm}^{0, \frac{1}{2}+}\left(S_{T}\right) \quad \text { and } \quad \phi_{ \pm}^{\mathrm{h}} \in X_{ \pm}^{\frac{1}{2}, \frac{1}{2}+}\left(S_{T}\right) .
$$

Moreover, (3.1) implies

$$
\psi_{ \pm} \in X_{ \pm}^{0,0}\left(S_{T}\right)
$$

so $(2.6)$ gives

$$
\psi_{ \pm}^{1} \in X_{ \pm}^{0,1}\left(S_{T}\right)
$$

Thus it only remains to show that the pair $\left(\Psi_{ \pm}, \Phi_{ \pm}\right)$satisfies (3.2). To this end we start with (3.1) and use the null structure and product estimates to successively improve the regularity.

Note that by the above, $\Psi_{ \pm}=\psi_{ \pm}-\psi_{ \pm}^{\mathrm{h}}-\psi_{ \pm}^{\mathrm{l}} \in X_{ \pm}^{0,0}\left(S_{T}\right)$, and a similar argument shows that $\Phi_{ \pm}=\phi_{ \pm}-\phi_{ \pm}^{\mathrm{h}} \in X_{ \pm}^{\frac{1}{2}, 0}$. 


\subsection{First estimate for $\Psi_{ \pm}$}

We first claim

$$
\Psi_{ \pm} \in X_{ \pm}^{-\frac{1}{2} \alpha, \alpha}\left(S_{T}\right) \text { for } \alpha \in[0,1]
$$

Indeed, using (2.6),

$$
\begin{aligned}
\left\|\Psi_{ \pm}\right\|_{X_{ \pm}^{-\frac{1}{2}, 1}\left(S_{T}\right)} \lesssim\|\phi \beta \psi\|_{X_{ \pm}^{-\frac{1}{2}, 0}\left(S_{T}\right)} & =\|\phi \beta \psi\|_{L_{t}^{2} H_{x}^{-\frac{1}{2}}\left(S_{T}\right)} \\
& \lesssim T^{\frac{1}{2}}\|\phi\|_{L_{t}^{\infty} H_{x}^{\frac{1}{2}}\left(S_{T}\right)}\|\psi\|_{L_{t}^{\infty} L_{x}^{2}\left(S_{T}\right)},
\end{aligned}
$$

where in the last line we used the product Sobolev inequality in the $x$-variable (see, e.g., the introduction in [4]). Interpolating $\Psi_{ \pm} \in X_{ \pm}^{-\frac{1}{2}, 1}\left(S_{T}\right)$ with $\Psi_{ \pm} \in$ $X_{ \pm}^{0,0}\left(S_{T}\right)$, we get $(3.3)$.

\subsection{First estimate for $\Phi_{ \pm}$}

We show that

$$
\Phi_{ \pm} \in X_{ \pm}^{\frac{1}{4}-\varepsilon, \frac{1}{2}+\varepsilon}\left(S_{T}\right)
$$
estimate

Using (3.3) with $\alpha=\frac{1}{4}+2 \varepsilon$ and (2.6), the claim reduces to the bilinear

$$
\left\|\left\langle\beta P_{ \pm_{1}}(D) \psi, P_{ \pm_{2}}(D) \psi^{\prime}\right\rangle\right\|_{X_{ \pm_{0}}^{-\frac{3}{4}-\varepsilon,-\frac{1}{2}+\varepsilon}} \lesssim\|\psi\|_{X_{ \pm_{1}}^{-\frac{1}{8}-\varepsilon, \frac{1}{4}+2 \varepsilon}}\left\|\psi^{\prime}\right\|_{X_{ \pm_{2}}^{-\frac{1}{8}-\varepsilon, \frac{1}{4}+2 \varepsilon}} .
$$

Without loss of generality, take $\pm_{1}=+, \pm_{2}= \pm$. By Plancherel and using (2.3) we reduce to

$$
I \lesssim\|u\|_{X_{+}^{-\frac{1}{8}-\varepsilon, \frac{1}{4}+2 \varepsilon}}\|v\|_{X_{ \pm}^{-\frac{1}{8}-\varepsilon, \frac{1}{4}+2 \varepsilon}}
$$

where

$$
I=\left\|\iint \frac{\theta(\eta, \pm(\eta-\xi))}{\langle\xi\rangle^{\frac{3}{4}+\varepsilon}\langle|\tau|-|\xi|\rangle^{\frac{1}{2}-\varepsilon}} \widehat{u}(\lambda, \eta) \widehat{v}(\lambda-\tau, \eta-\xi) d \lambda d \eta\right\|_{L_{\tau, \xi}^{2}} .
$$

Applying Lemma 2.2 with $a=\frac{1}{2}-\varepsilon$ and $b=c=\frac{1}{4}+2 \varepsilon$, and using also (2.5), the estimate for $I$ reduces to the following three estimates:

$$
\left\{\begin{array}{l}
\|u v\|_{H^{-\frac{3}{4}-\varepsilon, 0}} \lesssim\|u\|_{H^{\frac{3}{8}-2 \varepsilon, \frac{1}{4}+2 \varepsilon}}\|v\|_{H^{-\frac{1}{8}-\varepsilon, \frac{1}{4}+2 \varepsilon}}\|v\| v\left\|_{H^{-\frac{3}{4}-\varepsilon,-\frac{1}{2}+\varepsilon}} \lesssim\right\| u\left\|_{H^{\frac{1}{8}+\varepsilon, 0}}\right\| v \|_{H^{-\frac{1}{8}-\varepsilon, \frac{1}{4}+2 \varepsilon}} \\
\|u v\|_{H^{-\frac{3}{4}-\varepsilon,-\frac{1}{2}+\varepsilon}} \lesssim\|u\|_{H^{-\frac{1}{8}-\varepsilon, 0}}\|v\|_{H^{\frac{1}{8}+\varepsilon, \frac{1}{4}+2 \varepsilon}} .
\end{array}\right.
$$

All these hold by Theorem 2.3 (via duality for the last two), proving (3.4). Interpolating with $\Phi_{ \pm} \in X_{ \pm}^{\frac{1}{2}, 0}$ we get moreover

$$
\Phi_{ \pm} \in X_{ \pm}^{\frac{7}{16}-\frac{\varepsilon}{4}, \frac{1}{8}+\frac{\varepsilon}{4}}\left(S_{T}\right)
$$

which we now use to improve (3.3). 


\subsection{Second estimate for $\Psi_{ \pm}$}

We show that

$$
\Psi_{ \pm} \in X_{ \pm}^{-\frac{7}{32}, \frac{1}{2}+\varepsilon}\left(S_{T}\right)
$$

Applying (2.6) and using (3.3) (with $\alpha=\frac{1}{2}-2 \varepsilon$ ) and (3.6), the claim reduces to the bilinear estimate

$$
\left\|P_{ \pm_{2}}(D)\left(\phi \beta P_{ \pm_{1}}(D) \psi\right)\right\|_{X_{ \pm_{2}}^{-\frac{7}{32},-\frac{1}{2}+\varepsilon}} \lesssim\|\phi\|_{X_{ \pm_{0}}^{\frac{7}{16}-\frac{\varepsilon}{4}, \frac{1}{8}+\frac{\varepsilon}{4}}}\|\psi\|_{X_{ \pm_{1}}^{-\frac{1}{4}+\varepsilon, \frac{1}{2}-2 \varepsilon}} .
$$

Duality reduces this to

$$
\left\|\left\langle\beta P_{ \pm_{1}}(D) \psi, P_{ \pm_{2}}(D) \psi^{\prime}\right\rangle\right\|_{X_{ \pm_{0}}^{-\frac{7}{16}+\frac{\varepsilon}{4},-\frac{1}{8}-\frac{\varepsilon}{4}}} \lesssim\left\|\psi^{\prime}\right\|_{X_{ \pm_{1}}^{\frac{7}{32}, \frac{1}{2}-\varepsilon}}\|\psi\|_{X_{ \pm_{2}}^{-\frac{1}{4}+\varepsilon, \frac{1}{2}-2 \varepsilon}} .
$$

Taking again $\pm_{1}=+, \pm_{2}= \pm$ without loss of generality, applying Plancherel and using (2.3), we reduce further to

$$
J \lesssim\|u\|_{X_{+}^{\frac{7}{32}, \frac{1}{2}-\varepsilon}}\|v\|_{X_{ \pm}^{-\frac{1}{4}+\varepsilon, \frac{1}{2}-2 \varepsilon}}
$$

where

$$
J=\left\|\iint \frac{\theta(\eta, \pm(\eta-\xi))}{\langle\xi\rangle^{\frac{7}{16}-\frac{\varepsilon}{4}}\langle|\tau|-|\xi|\rangle^{\frac{1}{8}+\frac{\varepsilon}{4}}} \widehat{u}(\lambda, \eta) \widehat{v}(\lambda-\tau, \eta-\xi) d \lambda d \eta\right\|_{L_{\tau, \xi}^{2}},
$$

Applying Lemma 2.2 with $a=\frac{1}{2}, b=\frac{1}{2}-\varepsilon$ and $c=\frac{1}{2}-2 \varepsilon$, and using (2.5), we reduce to the following six estimates:

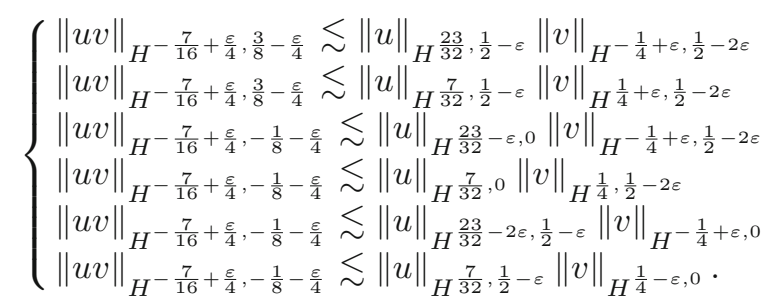

All these hold by Theorem 2.3 (via duality for the last four), proving (3.7). Interpolation with $\Psi_{ \pm} \in X_{ \pm}^{0,0}$ yields

$$
\Psi_{ \pm} \in X_{ \pm}^{-\frac{7}{64}, \frac{1}{4}+\frac{\varepsilon}{2}}\left(S_{T}\right)
$$

We now use this to improve (3.4).

\subsection{Second estimate for $\Phi_{ \pm}$}

We show that

$$
\Phi_{ \pm} \in X_{ \pm}^{\frac{9}{32}-\varepsilon, \frac{1}{2}+\varepsilon}\left(S_{T}\right)
$$

Applying (2.6) and using (3.9) we reduce to

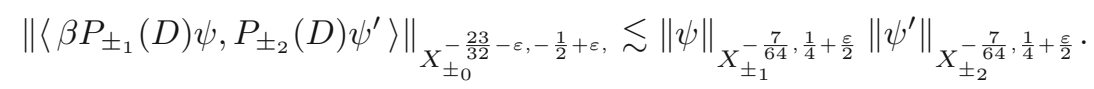


Proceeding as in Sect. 3.2, applying Lemma 2.2 with $a=\frac{1}{2}-\varepsilon$ and $b=c=\frac{1}{4}+\frac{\varepsilon}{2}$, and using (2.5), we further reduce to the following three estimates:

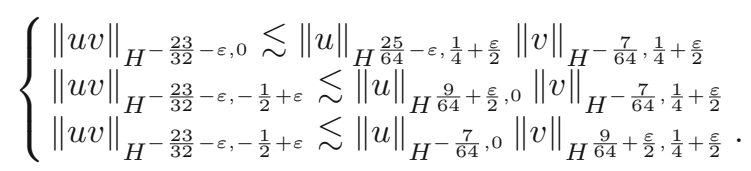

All these are seen to hold by Theorem 2.3 (via duality for the last two). Interpolating (3.10) with $\Phi_{ \pm} \in X_{ \pm}^{\frac{1}{2}, 0}$, we further get

$$
\Phi_{ \pm} \in X_{ \pm}^{\frac{25}{64}-\frac{\varepsilon}{2}, \frac{1}{4}+\frac{\varepsilon}{2}}\left(S_{T}\right) .
$$

\subsection{Third estimate for $\Psi_{ \pm}$}

We prove

$$
\Psi_{ \pm} \in X_{ \pm}^{-\frac{5}{32}-3 \varepsilon, \frac{1}{2}+\varepsilon}\left(S_{T}\right) .
$$

Applying (2.6) and using (3.7) and (3.12), the claim reduces (after duality) to

$$
\left\|\left\langle\beta P_{ \pm_{1}}(D) \psi, P_{ \pm_{2}}(D) \psi^{\prime}\right\rangle\right\|_{X_{ \pm_{0}}^{-\frac{25}{64}+\frac{\varepsilon}{2},-\frac{1}{4}-\frac{\varepsilon}{2}}} \lesssim\left\|\psi^{\prime}\right\|_{X_{ \pm_{1}}^{\frac{5}{32}+3 \varepsilon, \frac{1}{2}-\varepsilon}}\|\psi\|_{X_{ \pm_{2}}^{-\frac{7}{32}, \frac{1}{2}+\varepsilon}} .
$$

Proceeding as in Sect. 3.3, applying Lemma 2.2 with $a=\frac{1}{2}, b=\frac{1}{2}-\varepsilon$ and $b=\frac{1}{2}$, and using (2.5), we reduce to

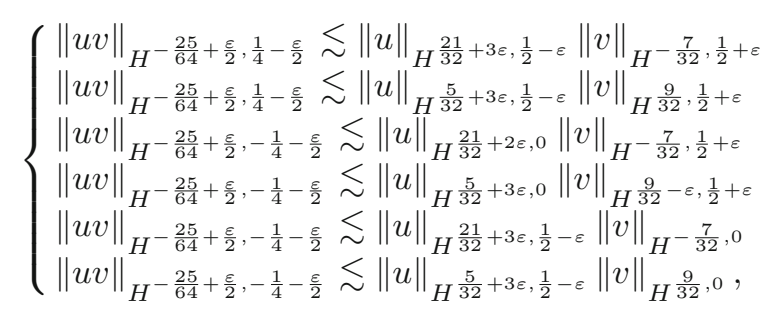

all of which hold by Theorem 2.3 (via duality for the last four).

\subsection{Third estimate for $\Phi_{ \pm}$}

We finally prove that

$$
\Phi_{ \pm} \in X_{ \pm}^{\frac{11}{32}-3 \varepsilon, \frac{1}{2}+\varepsilon}\left(S_{T}\right) .
$$

By (2.6) and (3.13) we reduce to

$$
\left\|\left\langle\beta P_{ \pm_{1}}(D) \psi, P_{ \pm_{2}}(D) \psi^{\prime}\right\rangle\right\|_{X_{ \pm_{0}}^{-\frac{21}{32}-3 \varepsilon,-\frac{1}{2}+\varepsilon}} \lesssim\|\psi\|_{X_{ \pm_{1}}^{-\frac{5}{32}-3 \varepsilon, \frac{1}{2}+\varepsilon}}\left\|\psi^{\prime}\right\|_{X_{ \pm_{2}}^{-\frac{5}{32}-3 \varepsilon, \frac{1}{2}+\varepsilon}} \text {. }
$$

Proceeding as in Sect. 3.2, applying Lemma 2.2 with $a=\frac{1}{2}-\varepsilon$ and $b=c=\frac{1}{2}$, the estimate reduces to

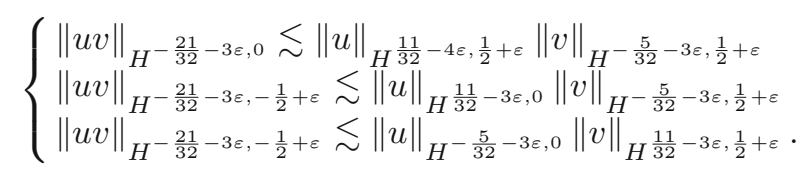


All these are true by Theorem 2.3.

This finishes the proof of Theorem 2.1.

\section{References}

[1] Bournaveas, N.: Low regularity solutions of the Dirac-Klein-Gordon equations in two space dimensions. Commun. Partial Differ. Equ. 26(7-8), 1345-1366 (2001)

[2] D'Ancona, P., Foschi, D., Selberg, S.: Null structure and almost optimal local regularity of the Dirac-Klein-Gordon system. J. EMS 9(4), 877-898 (2007)

[3] D'Ancona, P., Foschi, D., Selberg, S.: Local well-posedness below the charge norm for the Dirac-Klein-Gordon system in two space dimensions. J. Hyperbolic Differ. Equ. 2, 295-330 (2007)

[4] D’Ancona, P., Foschi, D., Selberg, S.: Product estimates for wave-Sobolev spaces in $2+1$ and $1+1$ dimensions. In: Nonlinear partial differential equations and hyperbolic wave phenomena. Contemporary Mathematics, vol. 526. American Mathematical Society, Providence pp. 125-150

[5] Grünrock, A., Pecher, H.: Global solutions for the Dirac-Klein-Gordon system in two space dimensions. Commun. Partial Differ. Equ. 35(1), 89-112 (2010)

[6] Pecher, H.: Unconditional well-posedness for the Dirac-Klein-Gordon system in two space dimensions. arXiv:1001.3065 (preprint)

[7] Zhou, Y.: Uniqueness of generalized solutions to nonlinear wave equations. Am. J. Math. 122(5), 939-965 (2000)

Sigmund Selberg and Achenef Tesfahun

Department of Mathematical Sciences

Norwegian University of Science and Technology

Alfred Getz' vei 1

7491 Trondheim

Norway

e-mail: sigmund.selberg@math.ntnu.no

A. Tesfahun

e-mail: tesfahun@math.ntnu.no

Received: 19 October 2011.

Accepted: 5 August 2012. 